\title{
G. Ranzi (a cura di), Dmitrij Šostakovič. Il grande compositore sovietico, Fondazione Mudima, Milano 2019, pp. 6ri.
}

Nella letteratura musicologica e russistica dedicata alla figura del compositore sovietico, questa pubblicazione si posiziona come aggiornamento degli studi svolti sino a questo momento in ambito prevalentemente italo-russo: la monografia definita in una recensione del musicologo Quirino Principe "il grande libro italiano per eccellenza su Dmitrij Dmitrievič Šostakovič", ovvero Šstakovič di Franco Pulcini (EDT, Torino 1988), e gli atti del convegno internazionale organizzato nel 2005 da Rosanna Giaquinta presso l'Università degli Studi di Udine, che ha visto protagonisti alcuni dei maggiori studiosi del compositore, come lo stesso Pulcini, Pauline Fairclough, Levon Hakopjan, Rosamund Bartlett e Manašir Jakubov (Dmitrij Šostakovič tra musica, letteratura e cinema, Olschki, Firenze 2008).

Questi testi costituiscono il prodotto più evidente di una ricerca che anche in Italia comincia a prendere piede, manifestandosi ancora come una sorta di 'fiume carsico', attraverso singoli contributi di entità minore, ma non per questo meno validi. In questo senso l'anniversario della rivoluzione del 1917 è stata una buona occasione, sfruttata in diversi convegni orientati allo studio delle arti performative: tra questi, il colloquio L'Ottobre delle arti svoltosi a Torino (Dipartimento di Studi Umanistici), dal quale è uscita l'omonima raccolta di atti curata da Giaime Alonge, Andrea Malvano e Armando Petrini (Academia University Press, Torino 2019), che contiene diversi contributi sulla produzione di Šostakovič - il saggio di Samuel Manzoni sulla Seconda Sinfonia - o al dibattito estetico-musicale che fa da sfondo alla produzione giovanile del compositore (Giust, Colombati, Randi). Manzoni, tra l'altro, aveva già pubblicato per LIM (2016) la traduzione di alcuni saggi di Sollertinskij, musicologo vicino al compositore, che molto influì sulla sua formazione (Musica e letteratura al tempo dell'Unione Sovietica, a cura di Samuel Manzoni, LIM, Lucca 2016). A questi si aggiungono le traduzioni italiane di testi già pubblicati in altre lingue, come Trascrivere la vita intera, Lettere 1923-1975 a cura di Elizabeth Wilson (introduzione di Enzo Restagno, traduzione di Laura Dusio, Il Saggiatore, Milano 2006), o Sinfonia di Leningrado di Brian Moynahan (traduzione di Claudia Manciocco, Il Saggiatore, Milano 2017).

Il libro qui recensito appare in linea con questo tipo di produzione, raccogliendo prevalentemente testi già pubblicati in russo, e apparsi in pubblicazioni come D.D. Šstakovič: Pro et contra (RGGU-RChGA, Moskva 2016) a cura di Levon Hakobian, o Šostakovič v Leningradskoj Konservatorii: I9I9-I930 a cura di L. G. Kovnackaja (3 voll., Kompozitor, Sankt-Peterburg 2013). Il volume consta di ventisette contributi organizzati in tre sezioni principali: la prima, intitolata PietrogradoLeningrado (pp. 45-I28), contiene due scritti di Anna Petrova dedicati alla città in cui il compositore 
nacque e si formò; la seconda, intitolata La musica (pp. 133-495) e legittimamente la più ampia, ospita voci moderne e storiche, da Ivan Sollertinskij a Levon Hakobian, da Luigi Pestalozza ed Eduardo De Filippo ad Anna Soudakova-Roccia, Dmitrij Braginskij, Manašir Jakubov, Roberta De Giorgi, Franco Pulcini, Oreste Bossini, Michail Pogarskij, Ol'ga Dombrovskaja, Evgenij Evtušenko, Dino Villatico, Luciano Alberti, Erasmo Valente e Valerij Voskobojnikov; la terza, intitolata Šostakovič e il suo tempo (pp. 499-6II), ha valore di bilancio memorialistico e al tempo stesso critico (con pagine di Hakopian, Šostakovič): affronta il problema del rapporto tra artista e potere che del compositore ha fatto un caso emblematico e include alcune note biografiche dello stesso compositore.

Aprono il volume due scritti introduttivi: Il grande compositore sovietico (pp. 9-16) di Gino Di Maggio, che è anche Presidente della Fondazione che ha voluto la pubblicazione, e "La mia musica non è mai come appare, si nasconde" (pp. 27-42) di Daniele Lombardi (1946-2018), compositore, pianista e artista visivo, già interprete di molte opere del futurismo musicale italiano e russo (autori come George Antheil, Leo Ornstein, Aleksandr Mosolov e Arthus Vincent Lourié), codirettore della collana "Fluid 39 ", di cui questo testo fa parte. Il primo contributo si fa carico di esplicitare il senso del titolo di questo "ennesimo libro su Dmitrij Šstakovič" (p. 9), dedicato a un compositore che "non è stato solo un grande musicista, ma anche una figura emblematica di artista e intellettuale del $\mathrm{xx}$ secolo, che ha vissuto da protagonista nell'arco della sua esistenza uno dei processi storici dell'umanità più complessi e drammatici. Ma anche più aperti alla speranza e al cambiamento di ogni tempo della storia umana" (p. 9). Il titolo vuole, in effetti, evidenziare il "senso di appartenenza alla sua comunità eticamente alto” (p. 9), testimoniato in primis dalla Sinfonia n. 7, detta 'leningradese' (alla quale è dedicato un saggio di Oreste Bossini, pp. 285-307, tra i pochi contributi originali): composta durante l'assedio della città ed ivi eseguita in segno di resistenza, fu trasmessa per radio per ricordare ai cittadini in guerra (e ai loro nemici) che "le cose nella nostra città procedono come sempre e che ognuno rimane al suo posto, malgrado la minaccia che pende sulla vita di Leningrado” (p. Io). Anche considerando questo aspetto di fedeltà del compositore al proprio Paese, agli occhi di chi scrive rimane tuttavia impossibile condividere l'assunto di Di Maggio, secondo il quale: "Dal 1917 al 1948, ossia per un lungo arco di tempo, la ricerca in Unione Sovietica è stata più volte oggetto di critiche dure, ma mai, o quasi mai, tassativamente 'vincolanti' per gli autori, che in modo evidente, nella loro grandissima maggioranza, hanno continuato ad attingere prevalentemente alle radici profonde della loro cultura musicale, soprattutto russa. È ampiamente documentabile, mi pare, che di fatto i grandi autori russo-sovietici di respiro internazionale non si sono mai sentiti vincolati da alcunché” (p. I4). Queste affermazioni, che esulano evidentemente dal contesto meramente musicale, si giustificano, se lo fanno, solo in quanto risposta provocatoria alla presa di posizione di certa musicologia anglofona, che per anni ha considerato la figura del compositore esclusivamente come 'vittima' di un sistema di potere autoritario, posizione che ha avuto come conseguenza diretta proprio il prevalere dell'aspetto simbolico della figura del musicista sulla qualità della sua produzione. In questo contesto, la figura di Šostakovič è stata a lungo strumentalizzata, ora in un senso, ora in senso diametralmente opposto: si pensi a Testimony di Solomon Volkov (Hamish Hamilton, London 1979), che nella sua faziosità (in senso decisamente anti-staliniano, ma politicamente scorretto in più direzioni) lascia ben poco spazio a quello che fu il vero mondo di Šostakovič, ovvero la musica.

Per fortuna, proprio la natura eterogenea dei saggi che compongono il volume basta a smorzare il carattere polemico dell'apertura, rendendo maggiore giustizia alla complessità dell'esperienza biografica e artistica del musicista, considerando sfaccettature diverse della sua produzione, dal teatro alla musica sinfonica, dalla musica da camera a quella applicata al cinema: si vedano, ad esempio, le prospettive divergenti gettate sull'opera giovanile Il naso, contenute negli scritti storici di Levon 
Hakobian (Il naso e la letteratura pietroburghese dell'assurdo, pp. I45-16I) e Luigi Pestalozza (Il naso di Dmitrij Šostakovič, pp. 163-179), corredati dal brevissimo saggio di Eduardo De Filippo dedicato al testo gogoliano (Il naso di Gogol', p. I8I), che ricorda l'approdo dell'opera šostakoviciana al teatro del Maggio musicale fiorentino, nel 1964; oppure si leggano le acute osservazioni, ancora di Hakopian, sul tema del difficile rapporto del compositore con le autorità staliniane e successive (Šostakovič e il potere sovietico: storia delle relazioni, pp. 499-533), che fanno da contraltare alle affermazioni sbilanciate di Di Maggio.

Ne nasce, quasi a dispetto dell'assunto iniziale, un insieme di sguardi che tutto sommato sembrano trovare un equilibrio nel loro complesso, e conferiscono all'aggettivo contenuto nel titolo connotazioni diverse, composite e ambivalenti, restituendo alla parola 'sovietico' molto del portato storico-culturale che viene invece escluso dall'accezione puramente politica che la parola assume quando viene intesa, come evidentemente fa Di Maggio, come sinonimo di 'stalinista'. A ciò si aggiungono aspetti più personali del compositore (cfr. Autobiografia, pp. 535-54I), che si integrano con il ricchissimo materiale iconografico raccolto da Anna Soudakova-Roccia presso diverse istituzioni russe (non ultimo l'Archivio Šostakovič di Mosca): ritratti del compositore e di personalità a lui vicine, pagine autografe, locandine di prime esecuzioni e bozzetti di scene e costumi, immagini storiche della città che fa da sfondo principale alla narrazione. Non foss'altro che per la qualità delle riproduzioni, queste immagini esulano dal ruolo di pura cornice ai testi, favorendo la ricostruzione del contesto, e costituendo per questo uno dei maggiori pregi del volume.

Nel suo complesso, questa antologia permette al lettore, se non di valutare i più recenti avanzamenti della ricerca dedicata al compositore e alla sua musica, sicuramente di conoscere quanto scritto da studiosi di calibro, e contribuisce a mettere in comunicazione due ambienti di ricerca quello italiano e quello russo - che in ambito musicale non hanno ancora trovato punti di contatto paragonabili a quelli degli studi letterari. 